\title{
Spin dynamics simulations of collective excitations in magnetic liquids
}

\author{
I.P.Omelyan ${ }^{1}$, I.M.Mryglod ${ }^{1,2}$, R.Folk ${ }^{2}$ \\ 1 Institute for Condensed Matter Physics \\ of the National Academy of Sciences of Ukraine, \\ 1 Svientsitskii Str., 79011 Lviv, Ukraine \\ 2 Institute for Theoretical Physics, University of Linz, A-4040 Linz, Austria
}

Received April 29, 2000

A novel approach is developed for computer simulation studies of dynamical properties of spin liquids. It is based on the Liouville operator formalism of Hamiltonian dynamics in conjunction with Suzuki-Trotter-like decompositions of exponential propagators. As a result, a whole set of symplectic time-reversible algorithms has been introduced for numerical integration of the equations of motion at the presence of both translational and spin degrees of freedom. It is shown that these algorithms can be used in actual simulations with much larger time steps than those inherent in standard predictor-corrector schemes. This has allowed one to perform direct quantitative measurements for spin-spin, spin-density and density-density dynamical structure factors of a Heisenberg ferrofluid model for the first time. It was established that like pure liquids the density spectrum can be expressed in terms of heat and sound modes, whereas like spin lattices in the ferromagnetic phase there exists one primary spin in the shape of spinspin dynamic structure factors describing the longitudinal and transverse spin fluctuations. As it was predicted in our previous paper [Mryglod I., Folk R. et al., Physica A277 (2000) 389] we found also that a secondary wave peak appears additionally in the longitudinal spin-spin dynamic structure factor. The frequency position of this peak coincides entirely with that for a sound mode reflecting the effect of the liquid subsystem on spin dynamics. The possibility of longitudinal spin wave propagation in magnetic liquids at sound frequency can be considered as a new effect which has yet to be tested experimentally.

Key words: Hejsenberg fluids, spin relaxation, collective modes, symplectic integration, Suzuki-Trotter decompositions

PACS: $02.60 . \mathrm{Cb}, 75.40 . \mathrm{Gb}, 75.50 . \mathrm{Mm}, 76.50 .+\mathrm{g}, 95.75 . \mathrm{Pq}$

\section{Introduction}

Magnetic systems have been the subject of numerous theoretical and computer experimental studies [1-6]. These studies were devoted mainly to describe static properties, such as phase transitions, scaling, and critical phenomena. The theoretical description of dynamic properties presents a more difficult problem which until 
now cannot be solved quantitatively even for the simplest magnetic models. For this reason, the method of molecular dynamics (MD) was used over recent years to investigate dynamic critical behaviour, dynamic scaling, and processes of spin relaxation. However, the MD simulations were restricted to lattice systems exclusively, in particular, to classical XY and Heisenberg models in $d=2$ as well as Heisenberg ferro- and antiferromagnets in $d=3$ dimensions [7-11].

The need in extensions of MD simulations to continuum disordered systems is motivated by a great variety of additional physical phenomena arising when both spin (orientational) and liquid (translational) degrees of freedom are taken into account. The existing theoretical investigations of magnetic liquid dynamics dealt to a great extent with phenomenological approaches [12-14]. Recently, the equations for time correlation functions and collective mode spectra of a Heisenberg ferrofluid model have been consequently derived using the method of nonequilibrium statistical operator $[15,16]$. As a result, a simple model of spin relaxation was considered, and the frequency matrix as well as the matrix of memory functions were calculated. However, this approach itself requires some additional information on the dynamic behaviour expressed in terms of correlation times. It is also worth mentioning that the main part of the theoretical results was obtained for a spin subsystem only and the mutual effect of the liquid subsystem on the spin dynamics has not been studied in detail.

In view of the afore said, computer experiments should be considered as the chief tool for quantitative studies of the dynamic properties. Nevertheless, there were no attempts known to simulate magnetic liquids within the MD approach. This can be explained by the absence of a suitable algorithm for solving the corresponding equations of motion. The traditional numerical methods [17] cannot be used for observations over a many-body system because of their high instability on MD scales of time. It was established for lattice systems that even standard predictor-corrector schemes appear to be inefficient because of very poor conservations of energy and spin lengths [18].

Obviously, an efficient algorithm for long-duration integrations should be stable, accurate, fast and easy to implement. There is only a limited group of integrators satisfying these requirements. Among them one can distinguish the velocity Verlet algorithm $[19,20]$ which allows us to obtain a high level of accuracy with minimal computational costs measured in terms of time-consuming force evaluations. However, the velocity Verlet and other similar schemes [20-22] were constructed to integrate translational motion without the presence of any orientational degrees of freedom. In our case the pattern is still more complicated since the translational positions and momenta are coupled with spin orientations in a characteristic way and, therefore, all these dynamical variables should be integrated simultaneously. This requires substantial revision of the liquid dynamic algorithms.

Quite recently, new spin dynamics algorithms have been proposed to simulate lattice systems [18]. They were constructed within the Suzuki-Trotter technique [23] using sublattice decompositions of spin dynamics. As was demonstrated, the sublattice decomposition algorithms unlike usual schemes exactly conserve individual spin 
lengths, exhibit good stability and energy conserving properties, and allow much larger time steps than predictor-corrector methods. The main problem with these algorithms lies in the fact that they are applicable only to spin systems when the decomposition on two or several noninteracting sublattices is possible. Such algorithms cannot be used for models with arbitrary spatial spin distributions and, thus, not for spin liquids.

In the current paper we develop the Suzuki-Trotter decomposition method and derive the desired spin liquid algorithms of different orders in the time step. The algorithms are tested in actual MD simulations on a Heisenberg ferrofluid model and compared with predictor-corrector schemes. The computations of spin-spin, spindensity and density-density time correlation functions are also carried out and the discussion on the surprising results obtained is added.

\section{Basic equations of motion}

Let us consider a classical fluid composed of $N$ magnetic particles of mass $m$ each containing continuous three-component spin $\mathbf{s}_{i}=\left(s_{i}^{x}, s_{i}^{y}, s_{i}^{z}\right)$ with the fixed length $\left|\mathbf{s}_{i}\right|=1$ for each site $i=1,2, \ldots, N$. A typical model Hamiltonian for such a system can be presented as

$$
H=\sum_{i=1}^{N} \frac{m \mathbf{v}_{i}^{2}}{2}+\sum_{i<j}^{N}\left(\varphi_{i j}-J_{i j}\left(s_{i}^{x} s_{j}^{x}+s_{i}^{y} s_{j}^{y}+\lambda s_{i}^{z} s_{j}^{z}\right)\right)-G \sum_{i=1}^{N} s_{i}^{z} .
$$

Here $\mathbf{v}_{i} \equiv \mathrm{d} \mathbf{r}_{i} / \mathrm{d} t$ and $\mathbf{r}_{i}$ are the translational velocity and position of particle $i$, respectively, $\varphi_{i j} \equiv \varphi\left(r_{i j}\right)$ is the liquid potential, $J_{i j} \equiv J\left(r_{i j}\right)$ denotes the exchange integral corresponding to a pair $(i, j)$ of spins with the interparticle separation $r_{i j}=\left|\mathbf{r}_{i}-\mathbf{r}_{j}\right|$, the exchange anisotropy parameter is denoted by $\lambda$, and $G$ is the intensity of an external spatially and timely homogeneous magnetic field $\mathbf{G} \equiv(0,0, G)$ directed along the $z$-th axis. Equation (1) represents, in fact, the isotropic $(\lambda=1)$ or anisotropic $(\lambda \neq 1)$ Heisenberg ferro- or the corresponding antiferro-fluid for $J_{i j}>0$ and $J_{i j}<0$, respectively. Although the main results which will be obtained in this paper are applicable for a larger class of Hamiltonians, we restrict ourselves to simplify notations to the basic model (1).

In the Liouville formulation of Hamiltonian dynamics, the equations of motion for the set $\boldsymbol{\rho} \equiv\left\{\mathbf{r}_{i}, \mathbf{v}_{i}, \mathbf{s}_{i}\right\}$ of microscopic variables are of the form

$$
\frac{\mathrm{d} \boldsymbol{\rho}}{\mathrm{d} t}=[\boldsymbol{\rho}, H] \equiv L \boldsymbol{\rho}(t),
$$

where

$$
L=\sum_{i=1}^{N}\left(\mathbf{v}_{i} \cdot \frac{\partial}{\partial \mathbf{r}_{i}}+\frac{\mathbf{f}_{i}}{m} \cdot \frac{\partial}{\partial \mathbf{v}_{i}}-\frac{1}{\hbar}\left[\left(\mathbf{g}_{i}+\mathbf{G}\right) \times \mathbf{s}_{i}\right] \cdot \frac{\partial}{\partial \mathbf{s}_{i}}\right) \equiv L_{\mathbf{r}}+L_{\mathbf{v}}+L_{\mathbf{s}}
$$

is the Liouville operator [15] of the system with

$$
\mathbf{f}_{i}=-\sum_{j(j \neq i)}^{N}\left[\frac{\mathrm{d} \varphi_{i j}}{\mathrm{~d} r_{i j}}-\frac{\mathrm{d} J_{i j}}{\mathrm{~d} r_{i j}}\left(s_{i}^{x} s_{j}^{x}+s_{i}^{y} s_{j}^{y}+\lambda s_{i}^{z} s_{j}^{z}\right)\right] \frac{\mathbf{r}_{i j}}{r_{i j}}
$$


being the translational force, and

$$
\mathbf{g}_{i} \equiv\left(g_{i}^{x}, g_{i}^{y}, g_{i}^{z}\right)=\sum_{j(j \neq i)}^{N} J_{i j}\left(s_{j}^{x}, s_{j}^{y}, \lambda s_{j}^{z}\right)
$$

the internal magnetic field. Note that the components $L_{\mathbf{r}}, L_{\mathbf{v}}$ and $L_{\mathbf{s}}$ act only on position, velocity and spin, respectively, and the quantum Poisson bracket [, ] was used to derive the expression for $L_{\mathbf{s}}$. If an initial configuration $\boldsymbol{\rho}(0)$ is determined, the time evolution of $\boldsymbol{\rho}(t)$ can be obtained numerically by integrating equation (2).

Taking into account the symmetries $\varphi_{i j}=\varphi_{j i}$ and $J_{i j}=J_{j i}$, it follows from equations (1) and (2) that the total energy $E \equiv H$ and the total momentum $\mathbf{P}=$ $m \sum_{i} \mathbf{v}_{i}$ of the system are integrals of motion, i.e. $\mathrm{d} E / \mathrm{d} t=0$ and $\mathrm{d} \mathbf{P} / \mathrm{d} t=0$. These symmetries also impose a conservation law for the magnetization $\mathbf{M}=\sum_{i} \mathbf{s}_{i}$ of the isotropic Heisenberg model at the absence of an external magnetic field. For the anisotropic case (when $\lambda \neq 1$ and/or $G \neq 0$ ) only the $z$-th component $M_{z}$ of $\mathbf{M}$ will not change in time. The structure of equation (2) requires the conservation of individual spin lengths as well. Moreover, it can be shown readily that our equations of motion are time reversible, i.e., they are invariant with respect to the set $t \rightarrow$ $-t,\left\{\mathbf{v}_{i}, \mathbf{s}_{i}\right\} \rightarrow\left\{-\mathbf{v}_{i},-\mathbf{s}_{i}\right\}$ of transformations. Besides, the exact solutions behave symplectically, i.e., the phase volume of $\left\{\mathbf{r}_{i}, \mathbf{v}_{i}\right\}$ will remain constant. Obviously, all the above properties cannot be perfectly reproduced during the integration within any numerical scheme. This is a typical situation in MD simulations of liquids. However, as we shall show in the next section, some of the integrals of motion as well as the time-reversibility and symplecticity can be maintained although the numerical trajectories are generated with a limited accuracy.

\section{Algorithms for numerical integration}

Within the Liouville exponential operator formalism, the solutions to the equations of motion (2) are cast in the form

$$
\boldsymbol{\rho}(t+h)=\mathrm{e}^{L h} \boldsymbol{\rho}(t)=\mathrm{e}^{\left(L_{\mathbf{r}}+L_{\mathbf{v}}+L_{\mathbf{s}}\right) h} \boldsymbol{\rho}(t),
$$

where $h$ denotes the time step. Since the exponential propagator $\mathrm{e}^{L h}$ cannot be evaluated exactly, it is necessary to introduce some approximations. These approximations should take into account the relatively small time step $h$ actually used in MD simulations.

\subsection{Second-order version}

Assuming for the moment that spin variables are frozen, i.e. letting $L_{\mathbf{s}} \rightarrow 0$, one comes to the usual (liquid-like) equations of motion. They can be solved in a quite efficient way applying the second-order VV integrator $[19,20]$ which is based on the Suzuki-Trotter (ST) formula $\mathrm{e}^{\left(L_{\mathbf{r}}+L_{\mathbf{v}}\right) h}=\mathrm{e}^{L_{\mathbf{v}} h / 2} \mathrm{e}^{L_{\mathbf{r}} h} \mathrm{e}^{L_{\mathbf{v}} h / 2}+\mathcal{O}\left(h^{3}\right)$. Unfreezing now the spin variables and treating the sum $L_{\mathbf{r}}+L_{\mathbf{s}}$ as one operator we obtain 
immediately: $\mathrm{e}^{\left(L_{\mathbf{r}}+L_{\mathbf{v}}+L_{\mathbf{s}}\right) h}=\mathrm{e}^{L_{\mathbf{v}} h / 2} \mathrm{e}^{\left(L_{\mathbf{r}}+L_{\mathbf{s}}\right) h} \mathrm{e}^{L_{\mathbf{v}} h / 2}+\mathcal{O}\left(h^{3}\right)$ (note that the ST formula is valid for arbitrary two operators). The spin-position subpropagator can further be decomposed in a similar way, $\mathrm{e}^{\left(L_{\mathbf{r}}+L_{\mathbf{s}}\right) h}=\mathrm{e}^{L_{\mathbf{r}} h / 2} \mathrm{e}^{L_{\mathbf{s}} h} \mathrm{e}^{L_{\mathbf{r}} h / 2}+\mathcal{O}\left(h^{3}\right)$, resulting into the full propagation

$$
\boldsymbol{\rho}(t+h)=\mathrm{e}^{L_{\mathbf{v}} \frac{h}{2}} \mathrm{e}^{L_{\mathbf{r}} \frac{h}{2}} \mathrm{e}^{L_{\mathbf{s}} h} \mathrm{e}^{L_{\mathbf{r}} \frac{h}{2}} \mathrm{e}^{L_{\mathbf{v}} \frac{h}{2}} \boldsymbol{\rho}(t)+\mathcal{O}\left(h^{3}\right) \equiv \mathbf{D}(t, h) \boldsymbol{\rho}(t)+\mathcal{O}\left(h^{3}\right) .
$$

Of course, other decompositions are also possible, but then the local fields $\mathbf{g}_{i}$ or/and forces $\mathbf{f}_{i}$ will need to be updated (the most time-consuming operations) more frequently, thus reducing the efficiency of the computations.

The main idea of the decompositions consists in obtaining subpropagators which can be evaluated analytically. As can be shown [20], the position $\mathrm{e}^{L_{\mathbf{r}} \tau}=\prod_{i} \mathrm{e}^{L_{\mathbf{r}_{i}} \tau}$ and velocity $\mathrm{e}^{L_{\mathbf{v}} \tau}=\prod_{i} \mathrm{e}^{L_{\mathbf{v}_{i}} \tau}$ propagation present shift operators, namely,

$$
\mathrm{e}^{L_{\mathbf{r}_{i}} \tau} \mathbf{r}_{i}=\mathbf{r}_{i}+\mathbf{v}_{i} \tau, \quad \mathrm{e}^{L_{\mathbf{v}_{i}} \tau} \mathbf{v}_{i}=\mathbf{v}_{i}+\frac{\mathbf{f}_{i}}{m} \tau
$$

where $L_{\mathbf{r}_{i}}=\mathbf{v}_{i} \cdot \partial / \partial \mathbf{r}_{i}, L_{\mathbf{v}_{i}}=\mathbf{f}_{i} / m \cdot \partial / \partial \mathbf{v}_{i}$, and the equalities $L_{\mathbf{r}_{i}} L_{\mathbf{r}_{j}}=L_{\mathbf{r}_{j}} L_{\mathbf{r}_{i}}$, $L_{\mathbf{v}_{i}} L_{\mathbf{v}_{j}}=L_{\mathbf{v}_{j}} L_{\mathbf{v}_{i}}$ have been used. It is worth emphasizing that the components $L_{\mathbf{r}}$ and $L_{\mathbf{v}}$ (as well as $L_{\mathbf{s}}$ ) do not commute between themselves. For this reason, the position and velocity shifts (8) must be performed in the rigorous order as specified by equation (7) and applied to actual current values of $\mathbf{r}_{i}$ and $\mathbf{v}_{i}$ within the time step.

The spin subdynamics is described in equation (7) by the exponential operator $\mathrm{e}^{L_{\mathbf{s}} h}$, where $L_{\mathbf{s}}=\sum_{i} L_{\mathbf{s}_{i}}$ and $L_{\mathbf{s}_{i}}=\left[\boldsymbol{\omega}_{i} \times \mathbf{s}_{i}\right] \cdot \partial / \partial \mathbf{s}_{i}$ with $\boldsymbol{\omega}_{i}=-\left(\mathbf{g}_{i}+\mathbf{G}\right) / \hbar$ being the local Larmor frequency. This operator has no simple explicit form, given that the internal magnetic field $\mathbf{g}_{i}$, acting on each particle, depends on the orientations of generally speaking (see equation (5)) all other spins of the system. In other words, it is itself time dependent and is, therefore, not known a priori. A possibility to obtain the explicit solutions lies in the following. Since all the partial components $L_{\mathbf{s}_{i}}$ do not commute each other, it is quite natural to find an ST-like decomposition for the whole set $(i=1, \ldots, N)$ of these noncommutative operators. Consecutively $(k=1, \ldots, N-1)$ dividing the subsets $(i=k, \ldots, N)$ into groups composed of only two operators $\left(L_{\mathbf{s}_{k}}\right.$ and $\left.\sum_{j=k+1}^{N} L_{\mathbf{s}_{j}}\right)$ and using the usual ST formula for them, one obtains

$$
\mathrm{e}^{L_{\mathbf{s}} h}=\mathrm{e}^{L_{\mathbf{s}_{1}} \frac{h}{2}} \ldots \mathrm{e}^{L_{\mathbf{s}_{N-1}} \frac{h}{2}} \mathrm{e}^{L_{\mathbf{s}_{N}} h} \mathrm{e}^{L_{\mathbf{s}_{N-1}} \frac{h}{2}} \ldots \mathrm{e}^{L_{\mathbf{s}_{1}} \frac{h}{2}}+\mathcal{O}\left(h^{3}\right) .
$$

Equation (9) constitutes an ST analogue for arbitrary number of operators and is accurate to the same order as that of the initially truncated (see equation (7)) terms $\mathcal{O}\left(h^{3}\right)$. Again, other $\mathcal{O}\left(h^{3}\right)$ decompositions can be introduced. However, only equation (9) will lead to a scheme with minimal number of local field recalculations.

The problem is now much simplified because, according to equation (9), each current value of $\mathbf{s}_{i}$ is updated spin by spin at a fixed instantaneous Larmor frequency $\boldsymbol{\omega}_{i}$, and this case allows analytical solutions. The result is

$$
\mathrm{e}^{L_{\mathbf{s}_{i}} \tau} \mathbf{s}_{i}(t)=\left(\mathbf{I}+\mathbf{W}_{i} \sin \left(\omega_{i} \tau\right)+\mathbf{W}_{i}^{2}\left(1-\cos \left(\omega_{i} \tau\right)\right)\right) \mathbf{s}_{i}(t) \equiv \boldsymbol{\Theta}_{i}(t, \tau) \mathbf{s}_{i}(t),
$$


where $\boldsymbol{\Theta}_{i}(t, \tau)$ denotes an orthonormal $\left(\boldsymbol{\Theta}^{+}=\mathbf{I}\right)$ matrix which rotates around axis $\boldsymbol{\omega}_{i}$ on angle $\omega_{i} \tau$, and $\mathbf{W}_{i}=\mathbf{W}\left(\boldsymbol{\omega}_{i}\right)$ is a skewsymmetric matrix $\left(\mathbf{W}_{\alpha \beta}=-\mathbf{W}_{\beta \alpha}\right)$ with the elements $\mathbf{W}_{X Y}=-\omega_{Z} / \omega, \mathbf{W}_{X Z}=\omega_{Y} / \omega, \mathbf{W}_{Y Z}=-\omega_{X} / \omega$. The trigonometric functions can be avoided in view of the fact that the above decompositions are only correct within $\mathcal{O}\left(h^{3}\right)$ uncertainty. It is, therefore, sufficient to replace them by rational counterparts, $\cos \xi=\left(1-\xi^{2} / 4\right) /\left(1+\xi^{2} / 4\right)+\mathcal{O}\left(\xi^{3}\right)$ and $\sin \xi=\xi /\left(1+\xi^{2} / 4\right)+$ $\mathcal{O}\left(\xi^{3}\right)$, which maintain the orthonormality of $\boldsymbol{\Theta}$. Then the spin rotation reduces to

$$
\mathrm{e}^{L_{\mathbf{s}_{i}} \tau} \mathbf{s}_{i}(t)=\frac{\mathbf{s}_{i}(t)+\left[\boldsymbol{\omega}_{i} \times \mathbf{s}_{i}(t)\right] \tau+\frac{1}{2}\left(\boldsymbol{\omega}_{i}\left(\boldsymbol{\omega}_{i} \cdot \mathbf{s}_{i}(t)\right)-\frac{1}{2}\left(\boldsymbol{\omega}_{i} \cdot \boldsymbol{\omega}_{i}\right) \mathbf{s}_{i}(t)\right) \tau^{2}}{1+\frac{1}{4}\left(\omega_{i} \tau\right)^{2}}+\mathcal{O}\left(\tau^{3}\right) .
$$

This completes our spin liquid dynamics (SLD) algorithm of the second-order.

It can be shown readily that the algorithm derived generates solutions which are time reversible and symplectic. Indeed, the initial propagator was decomposed (see equations (6), (7) and (9)) into subparts symmetrically, and, as a consequence, the final expressions for $\mathbf{r}_{i}, \mathbf{v}_{i}$ and $\mathbf{s}_{i}$ will be invariant with respect to the transformation $h \rightarrow-h$. Furthermore, simple shifts (8) applied separately in position and velocity space do not change the phase volume. These properties are very important for our purpose because, as is now well established [20,21], the stability of an algorithm normally follows from its time reversibility and symplecticity. Another nice property of the SLD integrator is its exact conservation of individual spin lengths (rotations given by equation (10) or (11) do not change the norm of vectors). This is crucial as well, since the condition $\left|\mathbf{s}_{i}\right|=$ const represents a major part of the definition for the model.

\subsection{Higher-order versions}

In many MD applications the accuracy achieved by second-order schemes is quite sufficient to obtain reliable results. However, if very high precision is required, higherorder algorithms may appear to be more efficient. The reason of this is that within these algorithms the truncation errors decrease more rapidly with decreasing of the step size. Applying very accurate MD integrators is especially important at phase transitions, for long-time correlated properties or when investigating derivatives of the thermodynamic functions.

A possibility to construct higher-order versions of the SLD algorithm lies in employing a so-called multiple staging technique [23]. This technique was used earlier for lattice spin dynamics [18] in the framework of usual ST decompositions. It was realized by us that the multiple staging integration can be performed with slight modifications within our approach as well. As a result, we have obtained the following expression

$$
\boldsymbol{\rho}(t+h)=\prod_{p=1}^{P} \mathbf{D}\left(t, \xi_{p} h\right) \boldsymbol{\rho}(t)+\mathcal{O}\left(h^{q+1}\right)
$$

for the $q$-th order propagation, where the coefficients $\xi_{p}$ are chosen at a given number $P$ to provide the highest possible value of $q$. For example, the fourth-order $(q=4)$ 
algorithm (SLD4) can be directly derived from equation (12) using $P=5$ and the coefficients $\xi_{1}=\xi_{2}=\xi_{4}=\xi_{5} \equiv \xi=1 /\left(4-4^{1 / 3}\right)$, and $\xi_{3}=1-4 \xi$. That is very interesting, these coefficients coincide with those found within the usual ST decomposition of exponential operators [23]. At $P=1$ and $\xi_{1}=1$, equation (12) reduces to the second order SLD algorithm.

It is worth remarking that within the higher-order versions $(q>2)$, the individual spin propagation must be carried out using exact rotations (10) rather than their second-order counterparts (11). Alternatively, the trigonometric functions can be avoided in equation (10) again, but then more precise rational approximations should be considered for them. In particular, the forth-order approximations are:

$$
\sin \xi=\frac{\xi-\frac{1}{12} \xi^{3}}{1+\frac{1}{12} \xi^{2}+\frac{1}{144} \xi^{4}}+\mathcal{O}\left(\xi^{5}\right), \quad \cos \xi=\frac{1-\frac{5}{12} \xi^{2}+\frac{1}{144} \xi^{4}}{1+\frac{1}{12} \xi^{2}+\frac{1}{144} \xi^{4}}+\mathcal{O}\left(\xi^{5}\right) .
$$

Expressions (13) ensure the orthonormality of $\boldsymbol{\Theta}$ and may be used within the SLD4 integration. Clearly, the fourth-order version SLD4 is time reversible since the multipliers $\xi_{p}$ enter symmetrically into equation (12). Moreover, it conserves the phase volume and individual spin lengths because the conservations are observed at each stage $p$. The corresponding versions with $q>4$ can also be explicitly introduced but they appear to be too demanding for practical calculations.

\subsection{Multiple time scale integration}

The existence of fast and slow processes in the system allows one to improve the efficiency of the decomposition integration using a multiple time scale (MTS) approach. The main idea of this approach is based on using a short time step for the rapidly varying strong forces, and a large time step for the smooth weak forces arising from the interactions at long interparticle distances. Then the most time-consuming slow forces will be recalculated less frequently saving significantly the computer time. Note that the MTS technique was originally constructed [22] to integrate pure liquid dynamics. Here we consider a question of how the MTS integration can be adapted to our SLD/SLD4 algorithms.

A way to realize this consists in splitting the liquid potential $\varphi_{i j}$ into fast $\left(r_{i j}<R\right)$ and slow $\left(r_{i j} \geqslant R\right)$ varying subparts which correspond to the repulsion $\left(\mathrm{d} \varphi_{i j} / \mathrm{d} r_{i j}<0\right)$ and attraction $\left(\mathrm{d} \varphi_{i j} / \mathrm{d} r_{i j} \geqslant 0\right)$ intervals of the interparticle distance $r_{i j}$. Then the total translational force (4) can be presented as the sum $\mathbf{f}_{i}=\mathbf{f}_{i}^{(\mathrm{f})}+\mathbf{f}_{i}^{(\mathrm{s})}$ of two terms,

$$
\begin{aligned}
\mathbf{f}_{i}^{(\mathrm{f})} & =-\sum_{j\left(j \neq i, r_{i j}<R\right)}^{N} \frac{\mathrm{d} \varphi_{i j}}{\mathrm{~d} r_{i j}} \frac{\mathbf{r}_{i j}}{r_{i j}}, \\
\mathbf{f}_{i}^{(\mathrm{s})} & =-\sum_{j\left(j \neq i, r_{i j} \geqslant R\right)}^{N} \frac{\mathrm{d} \varphi_{i j}}{\mathrm{~d} r_{i j}} \frac{\mathbf{r}_{i j}}{r_{i j}}+\sum_{j(j \neq i)}^{N}\left[\frac{\mathrm{d} J_{i j}}{\mathrm{~d} r_{i j}}\left(s_{i}^{x} s_{j}^{x}+s_{i}^{y} s_{j}^{y}+\lambda s_{i}^{z} s_{j}^{z}\right)\right] \frac{\mathbf{r}_{i j}}{r_{i j}},
\end{aligned}
$$

associated with the fast $\mathbf{f}_{i}^{(\mathrm{f})}$ and slow $\mathbf{f}_{i}^{(\mathrm{s})}$ varying subforces, respectively. This results into the separation $L_{\mathbf{v}_{i}}=L_{\mathbf{v}_{i}}^{(\mathrm{f})}+L_{\mathbf{v}_{i}}^{(\mathrm{s})}$ of the velocity part of the Liouville operator, 
where $L_{\mathbf{v}_{i}}^{(\mathrm{f})}=\frac{1}{m} \mathbf{f}_{i}^{(\mathrm{f})} \cdot \frac{\partial}{\partial \mathbf{v}_{i}}$ and $L_{\mathbf{v}_{i}}^{(\mathrm{s})}=\frac{1}{m} \mathbf{f}_{i}^{(\mathrm{s})} \cdot \frac{\partial}{\partial \mathbf{v}_{i}}$. Acting similarly as in the case of the derivation of ST-like decompositions (7) and (9), taking into account the above separation of $L_{\mathbf{v}_{i}}$, and collecting the fastest exponential operators inside the innermost cycles we obtain

$\boldsymbol{\rho}(t+h)=\mathrm{e}^{L_{\mathbf{v}}^{(\mathrm{s})} \frac{h}{2}}\left[\mathrm{e}^{L_{\mathbf{v}}^{(\mathrm{f})} \frac{h}{4 k}} \mathrm{e}^{L_{\mathbf{r}} \frac{h}{2 k}} \mathrm{e}^{L_{\mathbf{v}}^{(\mathrm{f})} \frac{h}{4 k}}\right]^{k} \mathrm{e}^{L_{\mathbf{s}} h}\left[\mathrm{e}^{L_{\mathbf{v}}^{(\mathrm{f})} \frac{h}{4 k}} \mathrm{e}^{L_{\mathbf{r}} \frac{h}{2 k}} \mathrm{e}^{L_{\mathbf{v}}^{(\mathrm{f})} \frac{h}{4 k}}\right]^{k} \mathrm{e}^{L_{\mathbf{v}}^{(\mathrm{s})} \frac{h}{2}} \boldsymbol{\rho}(t) \equiv \mathbf{D}^{\mathrm{mts}} \boldsymbol{\rho}(t)$,

where $\mathrm{e}^{L_{\mathbf{v}}^{(\mathrm{s})} \tau}=\prod_{i} \mathrm{e}^{L_{\mathbf{v}_{i}}^{(\mathrm{s})} \tau}$ and $\mathrm{e}^{L_{\mathbf{v}}^{(\mathrm{f})} \tau}=\prod_{i} \mathrm{e}^{L_{\mathbf{v}_{i}}^{(\mathrm{f})} \tau}$. During such a MTS integration the high energy collisions between particles at $r_{i j} \leqslant R$ are described more accurately by decreasing the time step to $h / k$, where $k>1$ is a parameter of the method.

Of course, the MTS integration requires additional computational efforts for performing $2 k$ recalculations of $\mathbf{f}_{i}^{(\mathrm{f})}$. But they will be compensated completely by using larger time steps $h$ being within the same accuracy. On the other hand, using the same time step for both the MTS (15) and usual (7) integrations, the precision of the calculations can be improved considerably with very little computational costs. This is so because the processor time needed to update $\mathbf{f}_{i}^{(\mathrm{f})}$ appears, as a rule, to be much smaller (at appropriately chosen values for the multistep parameter $k$ and $R$ ) than that to evaluate long-ranged term $\mathbf{f}_{i}^{(\mathrm{s})}$. Note that setting $R \rightarrow 0$ (then $\mathbf{f}_{i}^{(\mathrm{f})} \rightarrow 0$ and $\mathbf{f}_{i}^{(\mathrm{s})} \equiv \mathbf{f}_{i}$ ), the MTS spin dynamics propagation (15) reduces to the usual single time step SLD integration (7). It is also possible to implement the MTS technique within the SLD4 integration. Therefore it necessary to formally replace in equation (12) the propagator $\mathbf{D}(t, h)$ by their MTS analogue $\mathbf{D}^{\mathrm{mts}}(t, h)$.

\section{Applications to a Heisenberg ferrofluid model}

Our MD studies were based on an isotropic $(\lambda=1)$ Heisenberg ferrofluid model at the absence of an external magnetic field $(G=0)$. The Yukawa function [6] has been used to describe the spin-spin interactions. This function was truncated at $R_{\mathrm{c}}=2.5 \sigma$ and shifted to be zero at the truncation point to avoid force singularities,

$$
J(r)=\left\{\begin{array}{cc}
\epsilon\left[\frac{\sigma}{r} \exp \left(\frac{\sigma-r}{\sigma}\right)-\frac{\sigma}{R_{\mathrm{c}}} \exp \left(\frac{\sigma-R_{\mathrm{c}}}{\sigma}\right)\right], & r<R_{\mathrm{c}} \\
0 & r \geqslant R_{\mathrm{c}}
\end{array} .\right.
$$

The liquid subsystem was modelled by a soft-core potential [21],

$$
\varphi(r)=\left\{\begin{array}{cl}
4 \varepsilon\left[(\sigma / r)^{12}-(\sigma / r)^{6}\right]+\varepsilon, & r<2^{1 / 6} \sigma \equiv R \\
0 \quad, & r \geqslant R
\end{array} .\right.
$$

Here $\sigma$ denotes the diameter of particles, whereas $\epsilon$ and $\varepsilon$ are the intensities of the spin-spin and core-core interactions, respectively. The simulations were carried out in the cubic box (employing periodic boundary conditions) with $N=1000$ spins at a reduced density $n^{*}=\frac{N}{V} \sigma^{3}=0.6$, a reduced temperature $T^{*}=k_{\mathrm{B}} T / \epsilon=1.5<$ $T_{\mathrm{c}}^{*}$ (where $T_{\mathrm{c}}^{*} \approx 2.06$ is the critical temperature of the system [24]), a reduced 
core intensity $\varepsilon / \epsilon=1$, and a dynamical coupling parameter $d=\sigma(m \epsilon)^{1 / 2} / \hbar=2$. This parameter presents, in fact, the ratio $\tau_{\mathrm{tr}} / \tau_{\mathrm{s}}$, where $\tau_{\mathrm{tr}}=\sigma(m / \epsilon)^{1 / 2}$ and $\tau_{\mathrm{s}}=$ $\hbar / \epsilon$ are the characteristic time intervals of varying translational and spin variables, respectively.

As far as we shall investigate a ferrophase and deal with a microcanonical (NVES) ensemble, a non-zero magnetization of the system should be specified additionally. This quantity was taken from our separate single MC simulation [24], $\langle\mathbf{S}\rangle_{0} / N=0.6536 \pm 0.0001$, where \langle\rangle$_{0}$ denotes the canonical averaging (the finitesize effects were estimated to be less than 1\%). The MC simulation was performed using a biasing scheme [25] for sampling orientational degrees of freedom. Note that such a magnetization could be determined in MD simulations too, but then a canonical ensemble should be considered. This requires thermostatic forces and virtual magnetic fields to be included in the equations of motion, but this is beyond the scope of this paper.

All test runs were started from an identical well equilibrated configuration. The recalculation of local magnetic fields during spin subdynamics propagation (9) took approximately the same processor time as that of translational forces, spending in total $0.5 \mathrm{sec}$ per step on the Origin 2000 workstation. It is worth emphasizing that contrary to pure spin dynamics [18] (when auxiliary MC cycles are involved to generate configurations as initial conditions for equations of motion), the equilibration of our system can be performed within NVES MD simulations exclusively (where the initial values for $\mathbf{s}_{i}$ should be generated at the very beginning of the equilibration in order to fulfill the constraint $\left.\mathbf{S} \equiv\langle\mathbf{S}\rangle_{0}\right)$. This is possible due to the presence of energy exchange between the spin and liquid subsystems.

The MD results for the total energy $E^{*}=E / \epsilon$ and total spin $S$ as depending on the length $t$ of the simulations are presented in figure 1 (subsets (a)-(d) and (e)-(h), respectively). Four time steps, namely, $h^{*}=h / \tau_{\text {tr }}=0.00125,0.0025,0.005$ and 0.01 , were used to integrate the equations of motion within our decomposition SLD algorithm of the second order (equations (7)-(9) and (11)). For the purpose of comparison, the results obtained by us using a well established Adams-BashforthMoulton (ABM) predictor-corrector scheme [17] of the fourth-order are also included in figure 1 (dashed curves in subsets (a) and (b)). We mention that in this scheme the dynamical variables are first predicted, $\boldsymbol{\rho}(t+h)=\boldsymbol{\rho}(t)+[55 \dot{\boldsymbol{\rho}}(t)-59 \dot{\boldsymbol{\rho}}(t-$ $h)+37 \dot{\boldsymbol{\rho}}(t-2 h)-9 \dot{\boldsymbol{\rho}}(t-3 h)] h / 24+\mathcal{O}\left(h^{5}\right)$, and further iteratively corrected as $\boldsymbol{\rho}(t+h)=\boldsymbol{\rho}(t)+[9 \dot{\boldsymbol{\rho}}(t+h)+19 \dot{\boldsymbol{\rho}}(t)-5 \dot{\boldsymbol{\rho}}(t-h)+\dot{\boldsymbol{\rho}}(t-2 h)] h / 24+\mathcal{O}\left(h^{5}\right)$, where in our case $\dot{\boldsymbol{\rho}}=\left\{\mathbf{v}_{i}, \mathbf{f}_{i} / m,\left[\boldsymbol{\omega}_{i} \times \mathbf{s}_{i}\right]\right\}$. As can be seen from figure 1 (a), the ABM integrator exhibits similar equivalence in energy conservation at the smallest time step $h^{*}=0.00125$. However, this and other similar predictor-corrector schemes are neither reversible nor symplectic, and there is no evidence that the extra order obtained is relevant, since they have a very narrow region of stability and cannot be used for larger step sizes. This is demonstrated in figure 1 (b) for the ABM integrator (see dashed curve), where the total energy deviates systematically from the initial level leading to the fluctuations at the end of the run which exceed the fluctuations corresponding to the SLD integration (see solid curve) nearly 50 times! Note that 
very small step sizes are impractical because then too much expensive force and field evaluations should be done during the typical observation times. Moreover, the ABM scheme appears to be about 2 times slower even if one iteration only is applied within the corrector procedure.

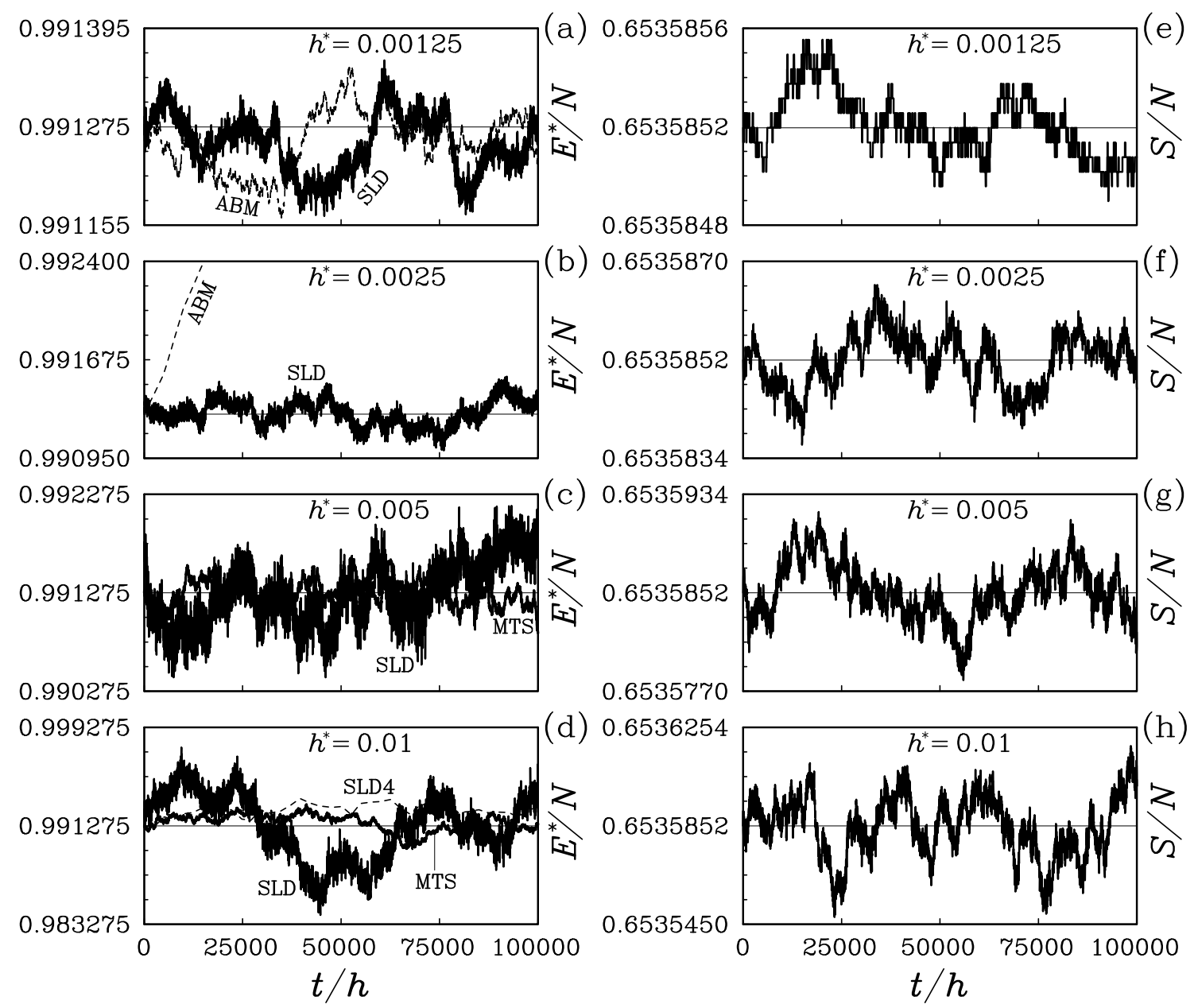

Figure 1. The reduced total energy $E^{*} / N$ (subsets (a)-(d)) and magnetization $S / N((\mathrm{e})-(\mathrm{h}))$ per spin as functions of the length $t / h$ of the simulations performed on a Heisenberg ferrofluid within the decomposition SLD (solid curves), ABM predictor-corrector (dashed curves in (a) and (b)), SLD4 (dashed curve in (e)), and MTS (solid curves in (c) and (d)) algorithms at four fixed time steps: $h^{*}=$ 0.00125 ((a), (e)); $0.0025((\mathrm{~b}),(\mathrm{f})) ; 0.005((\mathrm{c}),(\mathrm{g}))$; and $0.01((\mathrm{~d}),(\mathrm{h}))$. The initial levels $E^{*}(0) / N$ and $S(0) / N$ are plotted by the thin horizontal line.

No systematic drift in $E(t)$ and $S(t)$ was observed within our decomposition SLD algorithm at time steps up to $h^{*}=0.01$ over a length of $t / h=100000$. The precision of the algorithm was measured in terms of the ratio $\Gamma_{E}=(\langle(E(t)-$ $\left.\left.E(0))^{2}\right\rangle /\left\langle(U(t)-U(0))^{2}\right\rangle\right)^{1 / 2}$ of total $E$ and potential $U$ energy fluctuations. Taking into account that for our system $\left(\langle U(t)-U(0)\rangle^{2}\right)^{1 / 2} \approx 0.0335$, we have obtained: $\Gamma_{E} \approx 0.12 \%, 0.28 \%, 0.98 \%$ and $7.7 \%$ for the time steps $h^{*}=0.00125,0.0025,0.005$ 
and 0.01 , respectively. It is a common requirement, the ratio $\Gamma_{E}$ should not exceed one or two per cent to properly reproduce features of microcanonical ensembles. As we can see, time steps of $h^{*}<0.01$ satisfy this requirement and, thus, they can be used for precise calculations. The case $h^{*} \sim 0.01$ can also be acceptable when the precision is not so important, for example, in hybrid MC simulations [26], where only the time reversibility is required to satisfy detailed balance.

Note that the decomposition and ABM methods lead to the total momentum conservation within machine accuracy $\left(\sim 10^{-12}\right.$ in our program code). The reason is that all velocities are updated simultaneously and the interparticle forces can be evaluated exploiting the third Newton law. For similar reasons, the ABM integration maintains the total magnetization $\mathbf{S}$ (but it does not conserve individual spin lengths). During the decomposed integration the magnetization will not be conserved exactly. However, the fluctuations appear to be very small (see figure 1 (e)-(h)) and consist $\left\langle(\mathbf{S}(t)-\mathbf{S}(0))^{2}\right\rangle^{1 / 2} / N \approx 10^{-7}, 5 \cdot 10^{-7}, 2 \cdot 10^{-6}$ and $10^{-5}$ at $h^{*}=0.00125$, $0.0025,0.005$ and 0.01 , respectively.

An example on the total energy conservation obtained within the fourth-order SLD4 integration (equations (7)-(10), (12) and (13)) at $h^{*}=0.01 \equiv h_{\max }$ is shown in figure $1(\mathrm{~d})$. The energy fluctuations are decreased in this case approximately two times, i.e., $\Gamma_{E}^{(4)}\left(h_{\max }\right)=\Gamma_{E}\left(h_{\max }\right) / 2$. This compensates to some extent the additional computational efforts needed to evaluate high-order expressions (12). However, the SLD4 algorithm allows to reduce the magnetization deviations to a negligible small level which even for the greatest time step $h_{\max }=0.01$ does not exceed about $3 \cdot 10^{-9}$. Moreover, the time efficiency of the SLD4 algorithm will increase with decreasing of the step size, because the energy fluctuations tend to zero in this case as $\Gamma_{E}^{(4)}(h) \sim h^{4}$ at $h \rightarrow 0$, i.e., much faster than during the second-order SLD integration, where $\Gamma_{E}(h) \sim h^{2}$. The limiting value $\tilde{h}$ at which the integration will be performed with the same efficiency both for SLD and SLD4 algorithms can be estimated using the relation $\Gamma_{E}^{(4)}(\tilde{h})=\Gamma_{E}(\tilde{h} / P)$, where the fact that the five-stages SLD4 algorithm is in factor $P=5$ slower than has been used. Then in view of the explicit dependence of $\Gamma_{E}$ and $\Gamma_{E}^{(4)}$ on $h$ and the above boundary condition $\Gamma_{E}^{(4)}\left(h_{\max }\right)=\Gamma_{E}\left(h_{\max }\right) / 2$ one obtains that $\tilde{h}=h_{\max } \sqrt{2} / P \approx 0.003$. Therefore, the forth-order version SLD4 can be recommended if the total energy should be conserved with the precision $\Gamma_{E}(\tilde{h} / P)=\Gamma_{E}\left(h_{\max }\right)\left(\tilde{h} /\left(h_{\max } P\right)\right)^{2}=\Gamma_{E}\left(h_{\max }\right) 2 / P^{4} \approx 0.025 \%$ or better.

Finally, the function $E(t)$ corresponding to the MTS integration (equations (9), (11) and (15)) is plotted in figure 1 (c) and (d). The multistep parameter $k$ (appearing in equation (15)) was chosen to be equal to 3 . As can be seen, the MTS algorithm conserves the total energy more accurately than the usual SLD integrator. For instance at $h^{*}=h_{\max }$, the relative energy fluctuations are reduced in factor four, i.e., $\Gamma_{E}^{\operatorname{mts}}\left(h_{\max }\right)=\Gamma_{E}\left(h_{\max }\right) / 4$. To achieve such a reduction within the usual scheme, it is necessary to decrease the step size $h$ two times, and thus to increase the processor time by $100 \%$ for covering the fixed interval of integration. Within the MTS algorithm, the processor time needed to perform additional $2 k$ evaluations of fast varying forces consists of $45 \%$ only on the total computational time. Note that the fast varying forces $\mathbf{f}_{i}^{(\mathrm{f})}$ were updated in our case in $\sim\left(R_{\mathrm{c}} / R\right)^{3}=\left(2.5 / 2^{(1 / 6)}\right)^{3} \approx 11$ 
times faster than the long-ranged terms $\mathbf{f}_{i}^{(\mathrm{s})}$. The efficiency of the MTS integration will further increase (for larger systems) with increasing of the ratio $R_{\mathrm{c}} / R$.

\section{Investigation of collective excitations}

The dynamic behaviour of the Heisenberg fluid was investigated by measuring the spectra $F(k, \omega)=\frac{1}{2 \pi} \int_{-\infty}^{\infty} F(k, t) \mathrm{e}^{-\mathrm{i} \omega t} \mathrm{~d} t$ of collective density-density $F_{\text {nn }}(k, t)$, spin-spin $F_{\mathrm{ss}}^{\mathrm{L}, \mathrm{T}}(k, t)$, and spin-density $F_{\mathrm{sn}}^{\mathrm{L}}(k, t)$ time correlation functions,

$$
\begin{aligned}
F_{\mathrm{nn}}(k, t) & =\frac{1}{N}\left\langle\sum_{i, j}^{N} \mathrm{e}^{\mathrm{i} \mathbf{k} \cdot\left(\mathbf{r}_{i}(0)-\mathbf{r}_{j}(t)\right)}\right\rangle, \\
F_{\mathrm{ss}}^{\mathrm{L}, \mathrm{T}}(k, t) & =\frac{1}{N}\left\langle\sum_{i, j}^{N} \mathrm{~s}_{i}^{\mathrm{L}, \mathrm{T}}(0) \cdot \mathrm{s}_{j}^{\mathrm{L}, \mathrm{T}}(t) \mathrm{e}^{\mathrm{i} k \cdot\left(\mathbf{r}_{i}(0)-\mathbf{r}_{j}(t)\right)}\right\rangle, \\
F_{\mathrm{sn}}^{\mathrm{L}}(k, t) & =\frac{1}{N}\left\langle\sum_{i, j}^{N} \mathrm{~s}_{i}^{\mathrm{L}}(0) \mathrm{e}^{\mathrm{i} \mathbf{k} \cdot\left(\mathbf{r}_{i}(0)-\mathbf{r}_{j}(t)\right)}\right\rangle \equiv F_{\mathrm{ns}}^{\mathrm{L}}(k, t) .
\end{aligned}
$$

Note that the superscripts (L) and (T) refer to the longitudinal and transverse components of $\mathbf{s}_{i}$ with respect to the magnetization vector $\mathbf{S}$ rather than with respect to $\mathbf{k}$. The time correlation functions (18) were calculated using our SLD algorithm at $h^{*}=0.005$, and the microcanonical averaging \langle\rangle was taken over a length of 100 000 steps. This length is sufficient for the highest frequency resolution of $F(k, \omega)$. Ten independent runs with different initial equilibrium configurations were carried out to reduce the statistical noise.

The wavevector-dependent correlation functions $F_{\mathrm{nn}}(k), F_{\mathrm{sn}}^{\mathrm{L}}(k), F_{\mathrm{ss}}^{\mathrm{T}}(k)$, and $F_{\mathrm{ss}}^{\mathrm{L}}(k)$ obtained in the static limit $(t=0)$ are shown in figure 2 . The density-density structure factor $F_{\mathrm{nn}}(k)$ is related to a binary distribution function of magnetic particles averaged over their spin orientations. At intermediate and large wavevector values of $k$ this factor has a similar shape as that corresponding to pure liquid systems [27]. However, at small wavevectors we can observe deviations from the liquid behaviour. For example, the function $F_{\mathrm{nn}}(k)$ has an additional minimum at $k \sigma \sim 1.5$ (see subset (a)) that is caused by the existence of spin-spin interactions. The spindensity structure factor $F_{\mathrm{sn}}^{\mathrm{L}}(k)$ varies on wavevector like $F_{\mathrm{nn}}(k)$, but it appears to be shifted down at not very small $k$. This is so because in the infinite-wavevector regime $k \rightarrow \infty$ the function $F_{\mathrm{sn}}^{\mathrm{L}}(k)$ should tend to the magnetization $\mathbf{S} / N=0.6536$ of the system which cannot exceed unity, whereas $\lim _{k \rightarrow \infty} F_{\mathrm{nn}}(k)=1$ by definition.

At the same time, the transverse $F_{\mathrm{ss}}^{\mathrm{T}}(k)$ and longitudinal $F_{\mathrm{ss}}^{\mathrm{L}}(k)$ components of the spin-spin static correlation function differ between themselves in a characteristic way (see subset (b) of figure 2). While the transverse function tends to infinity at $k \rightarrow 0$ (this manifests itself the presence of Goldstone modes), the longitudinal component in this regime accepts finite values related to the magnetic susceptibility of the system. Note that the difference between $F_{\mathrm{ss}}^{\mathrm{T}}(k)$ and $F_{\mathrm{ss}}^{\mathrm{L}}(k)$ is due to the fact that we are investigating a ferrophase. In the case of a paraphase when the 

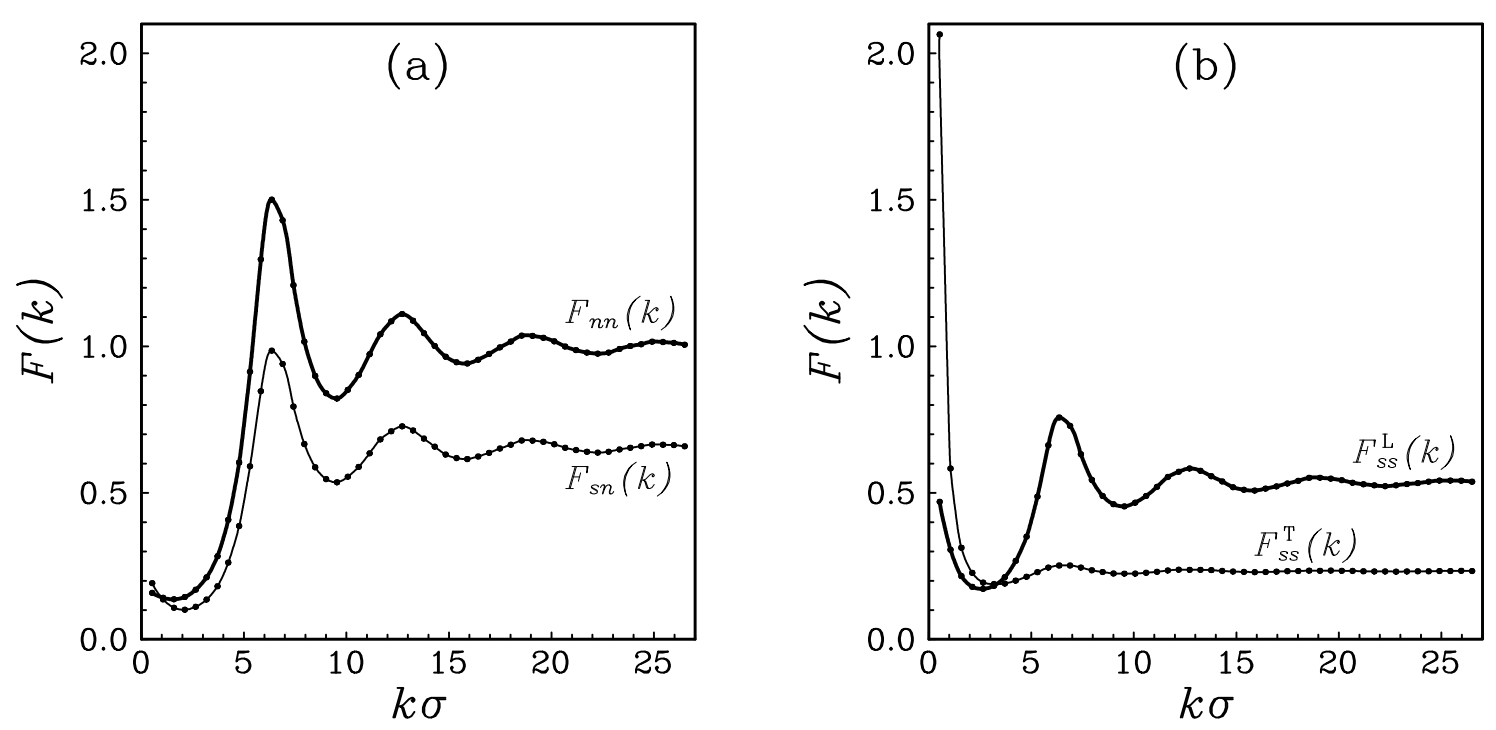

Figure 2. The static density-density $F_{\mathrm{nn}}(k)$ and spin-density $F_{\mathrm{sn}}^{\mathrm{L}}(k)($ subset (a)) as well as transverse $F_{\mathrm{sS}}^{\mathrm{T}}(k)$ and longitudinal $F_{\mathrm{sS}}^{\mathrm{L}}(k)$ spin-spin (subset (b)) correlation functions of a Heisenberg ferrofluid as depending on wavenumber.

magnetization is equal to zero (at the absence of an external field), these functions will be identical.

The density-density $F_{\mathrm{nn}}(k, \omega)$ and longitudinal spin-density $F_{\mathrm{sn}}^{\mathrm{L}}(k, \omega)$ dynamic structure factors are plotted in figures 3 and 4 , respectively. We have used a nondimensional presentation, $F^{*}(k, \omega)=F(k, \omega) / \tau_{\mathrm{tr}}$, for all the spectral functions with $\omega^{*}=\omega \tau_{\text {tr }}$ and $k^{*}=k / k_{\min }$, where $k_{\min }=2 \pi / V^{1 / 3} \approx 0.53 / \sigma$ is the smallest accessible wavevector. The peak observed in $F_{\mathrm{nn}}(k, \omega)$ at non-zero frequency $\omega=\omega_{\mathrm{s}}(k) \neq 0$ for each wavevector value $k \neq 0$ should be associated with a propagator sound mode well established for liquid systems $[27,28]$. This peak is especially visible in subset (a) of figure 1 corresponding to the smallest wavevector $k=k_{\min }$. At the same time, a maximum of $F_{\text {nn }}(k, \omega)$ at $\omega=0$ represents the well-known diffusive heat mode. Therefore, like pure liquids the density spectrum of the Heisenberg ferrofluid can be expressed in terms of one heat and two complex-conjugate sound modes with the dispersion $\omega_{\mathrm{s}}(k)$. The spin-density function $F_{\mathrm{sn}}^{\mathrm{L}}(k, \omega)$ behaves similar to $F_{\mathrm{nn}}(k, \omega)$. Note that the transverse component $F_{\mathrm{sn}}^{\mathrm{T}}(k, \omega)$ vanishes for arbitrary values of $k$ and $\omega$ because of $\left\langle\mathbf{s}_{i}^{\mathrm{T}}\right\rangle=0$.

Consider, finally, the process of spin relaxation described by the wavevector- and frequency-dependent transverse $F_{\mathrm{ss}}^{\mathrm{T}}(k, \omega)$ and longitudinal $F_{\mathrm{ss}}^{\mathrm{L}}(k, \omega)$ parts of the spinspin correlation function. These functions are shown in figures 5 and 6 , respectively. For the transverse function $F_{\mathrm{ss}}^{\mathrm{T}}(k, \omega)$ we have identified one peak at $\omega=\omega_{\mathrm{T}}^{*}(k)$ for each wavevector $k$ considered. This peak is very sharp at small $k$ (see subset (a) of figure 5) and shifts to the right with increasing $k$ (see subsets (b), (c) and (d)). Like lattice systems, such a quasiparticle behaviour should be associated with the existence of transverse spin waves in the Heisenberg ferrofluid. Up to three maxima were observed (see figure 6) for the longitudinal function $F_{\mathrm{ss}}^{\mathrm{L}}(k, \omega)$. In particular at 


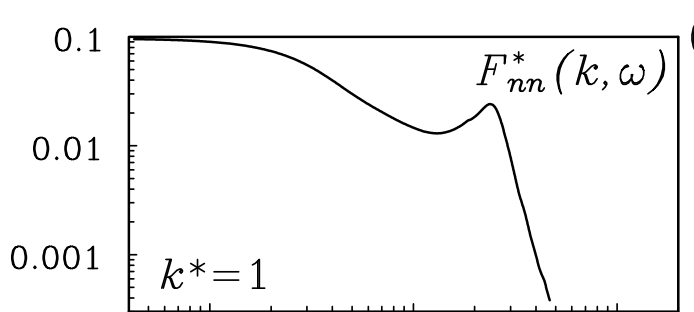

(a)

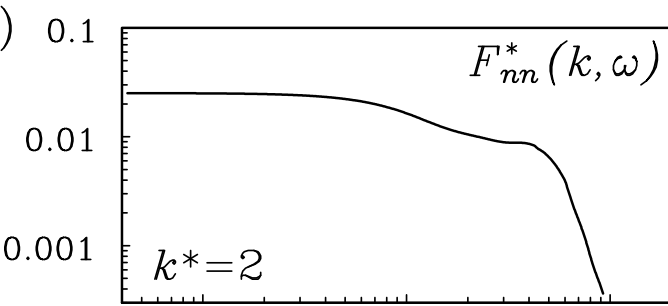

(b)

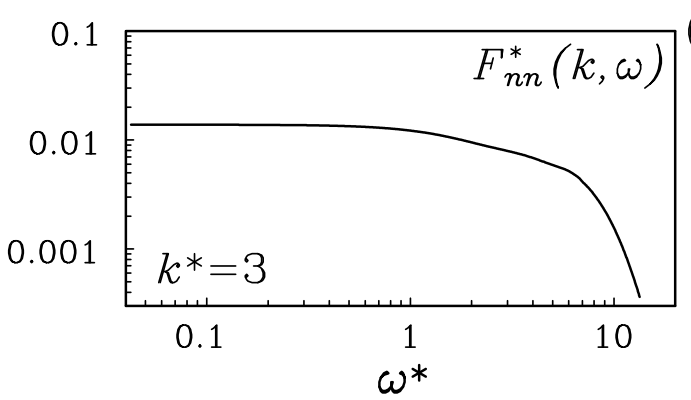

(c)

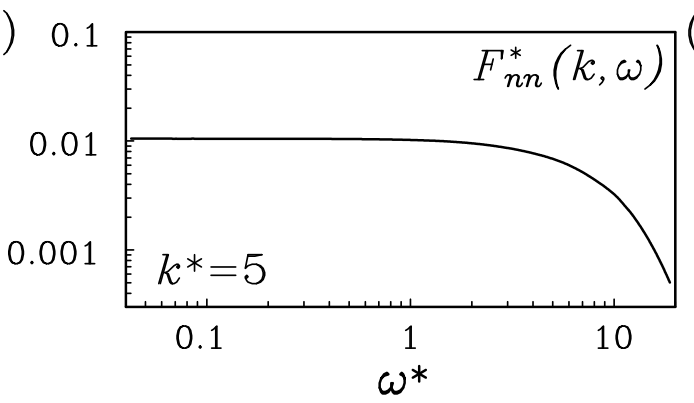

(d)

Figure 3. The density-density dynamic structure factor of a Heisenberg ferrofluid as a function of reduced frequency $\omega^{*}$ at four fixed values of reduced wavenumbers: $k^{*}=1(\operatorname{subset}(\mathrm{a})), k^{*}=2(\mathrm{~b}), k^{*}=3(\mathrm{c})$, and $k^{*}=5(\mathrm{~d})$.
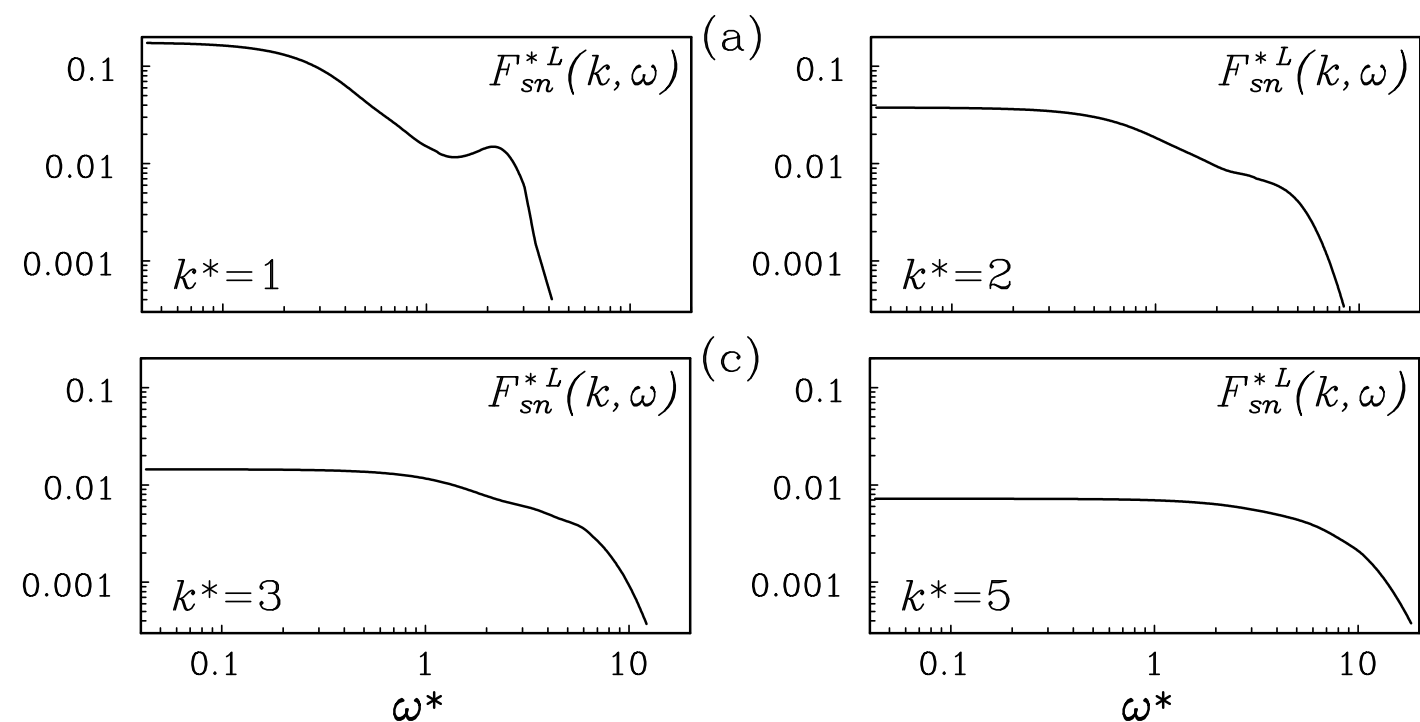

(c)

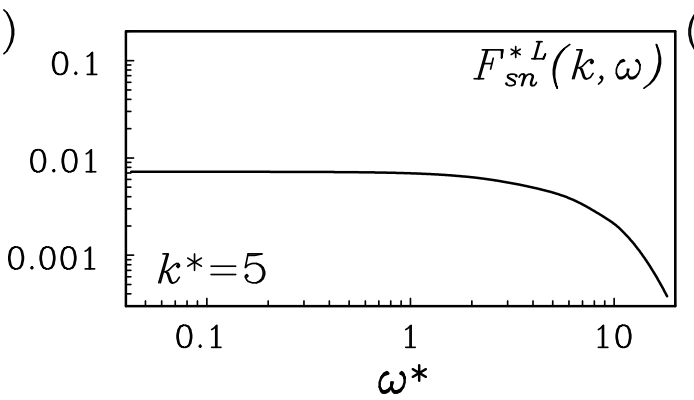

Figure 4. The spin-density dynamic structure factor of a Heisenberg ferrofluid as a function of reduced frequency $\omega^{*}$ at four fixed values of reduced wavenumbers: $k^{*}=1(\operatorname{subset}(\mathrm{a})), k^{*}=2(\mathrm{~b}), k^{*}=3(\mathrm{c})$, and $k^{*}=5(\mathrm{~d})$. 


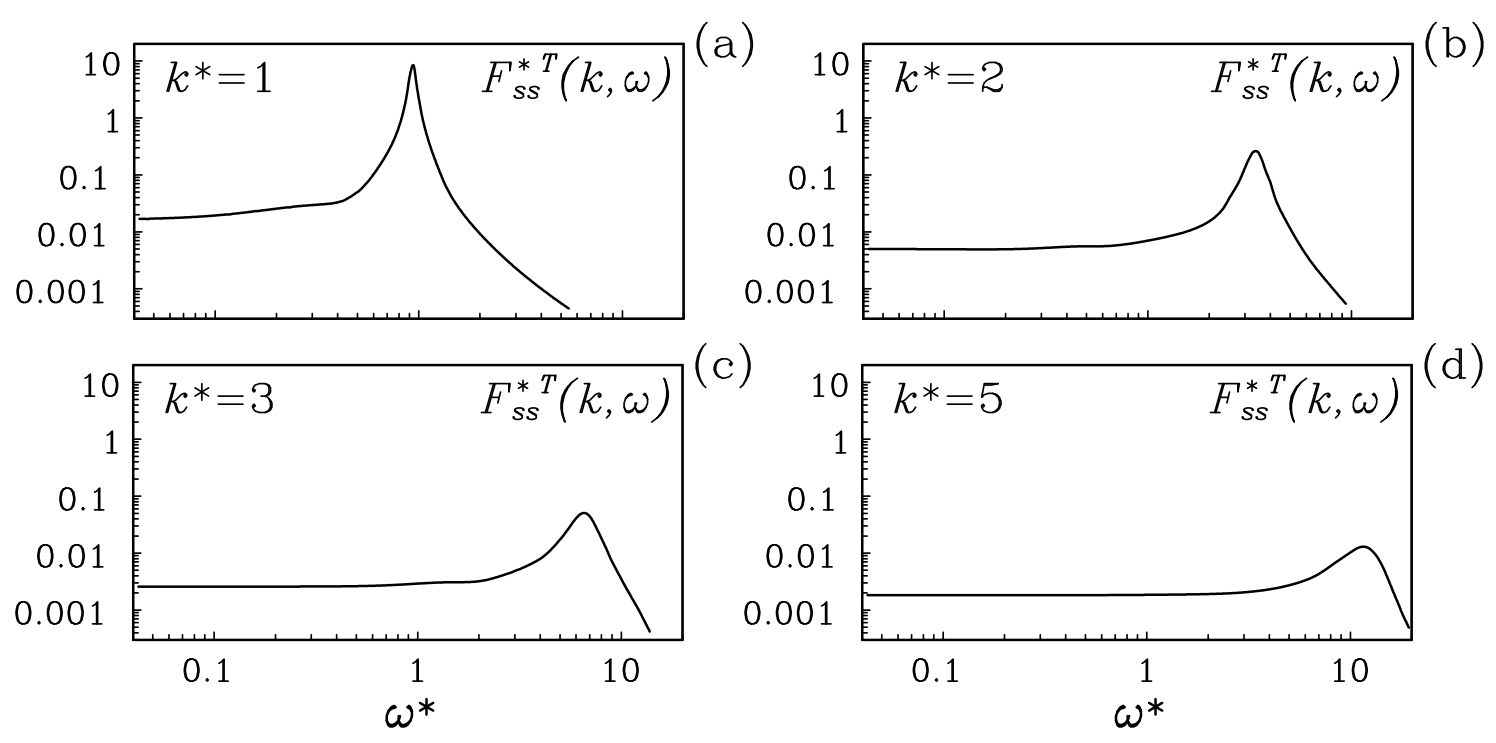

Figure 5. The transverse spin-spin dynamic structure factor of a Heisenberg ferrofluid as a function of reduced frequency $\omega^{*}$ at four fixed values of reduced wavenumbers: $k^{*}=1$ (subset (a)), $k^{*}=2(\mathrm{~b}), k^{*}=3(\mathrm{c})$, and $k^{*}=5(\mathrm{~d})$.
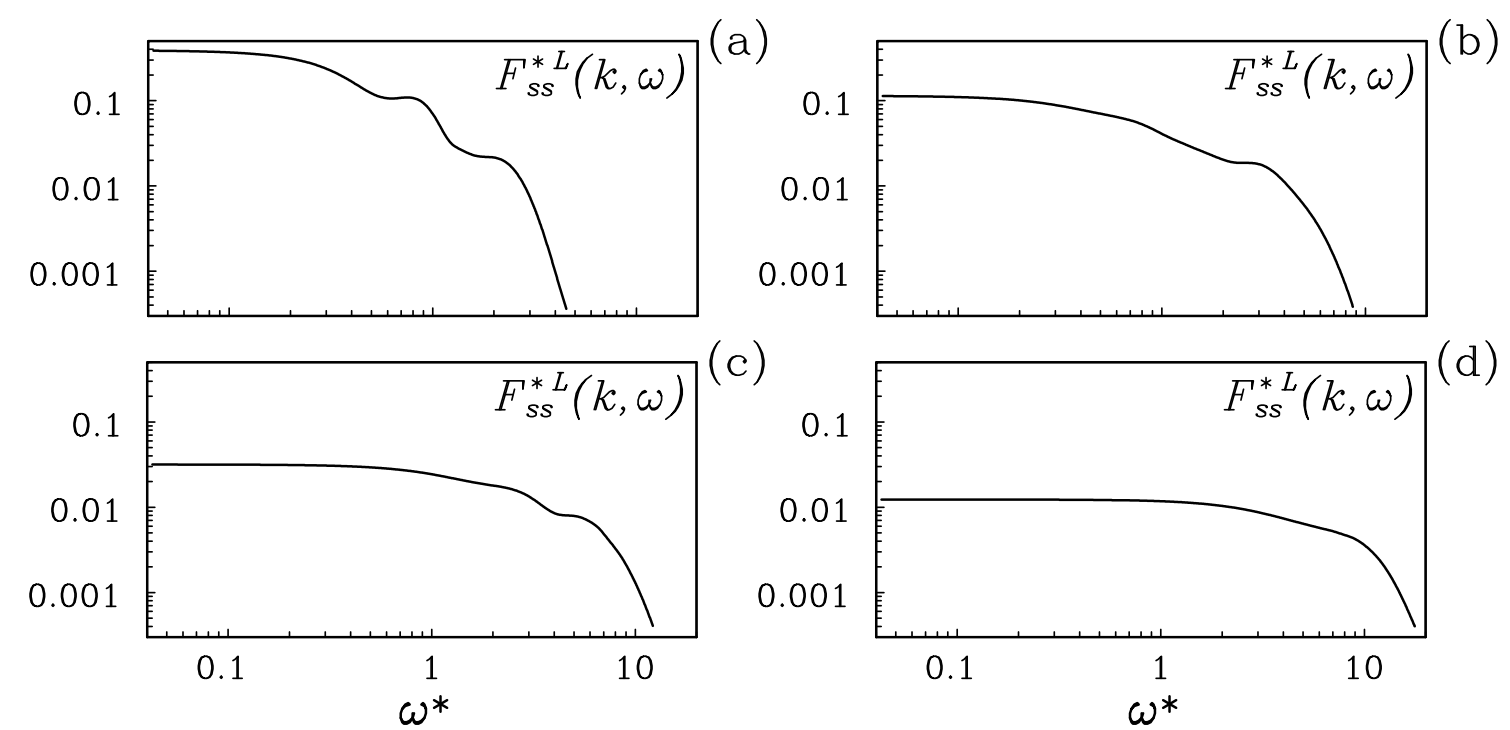

Figure 6. The longitudinal spin-spin dynamic structure factor of a Heisenberg ferrofluid as a function of reduced frequency $\omega^{*}$ at four fixed values of reduced wavenumbers: $k^{*}=1$ (subset (a)), $k^{*}=2(\mathrm{~b}), k^{*}=3(\mathrm{c})$, and $k^{*}=5(\mathrm{~d})$. 
$k^{*}=1$, the frequency positions for these maxima are: $\omega_{\mathrm{L}}^{*(1)}=0, \omega_{\mathrm{L}}^{*(2)} \approx \omega_{\mathrm{T}}^{*} \approx 1$ and $\omega_{\mathrm{L}}^{*(3)} \approx \omega_{\mathrm{s}}^{*} \approx 2.37$. While the first maximum at $\omega=0$ corresponds to pure diffusive relaxation processes, the position of the second maximum coincides with that of the transverse spin wave peak, indicating the possibility for propagation of longitudinal spin waves as well (which however are dumped more rapidly). The origin of the third maximum can be explained by direct effect of the liquid subsystem on spin relaxation, since its position coincides completely with the sound mode peak in the density spectrum.

In general, the results obtained for dynamic structure factors are in good agreement with predictions of [29]. The additional possibility of longitudinal spin wave propagation in magnetic liquids at sound frequency can be considered as a new effect which has yet to be quantitatively described in theory as well as observed experimentally.

\section{Conclusion}

Starting from the Liouville operator formalism in tandem with Suzuki-Trotterlike decompositions of exponential operators, we have devised and tested a class of new algorithms for numerical integration of the equations of motion at the presence of both liquid and spin subsystems. The greatest advantages of the new algorithms over standard predictor-corrector schemes can be summarized as follows: (i) symplecticity and time-reversibility, (ii) exact conservation of individual spin lengths, (iii) explicitness (no iteration), (iv) much more accuracy in the total energy conservation. Moreover, their excellent stability makes it possible to apply much larger time steps that may lead, therefore, to a substantial speedup of MD simulations on magnetic liquids.

As an example of an interesting physical system we have studied a three-dimensional isotropic Heisenberg ferrofluid model. Using the algorithms proposed has allowed one to determine quantitatively the density-density, spin-spin and spin-density dynamic structure factors for the first time. As a result, we have shown that like pure liquid systems the density spectrum can be described by one diffusive heat and two propagative sound modes. At the same time similar to spin lattices, the propagative transverse and longitudinal spin excitations are made of one primary wave peak at each wavevector. In the spectrum of longitudinal spin excitations, one secondary wave peak has been predicted additionally for the Heisenberg ferrofluid. It has been identified that the frequency position of this peak is identical to that of a sound mode. This result evidently follows from the effect of the liquid subsystem on spin dynamics.

The presence of a secondary wave peak in magnetic liquids indicates the possibility of longitudinal spin wave propagation at sound frequency. Such propagation should be considered as a new effect which has yet to be directly tested experimentally. The question of how to describe quantitatively this effect in theory will be a subject of our further studies. 


\section{Acknowledgements}

Part of this work was supported by the Fonds zur Förderung der wissenschaftlichen Forschung under Project No. P12422-TPH.

\section{References}

1. Amit D.J. Field Theory, the Renormalization Group, and Critical Phenomena. New York, McGraw-Hill, 1978.

2. Handrich K., Kobe S. Amorphe Ferro- und Ferrimagnetika. Berlin, Akademika-Verlag, 1980.

3. Parisi G. Statistical Field Theory. Wokingham, Addison-Wesley, 1988.

4. Lomba E., Weis J.J., Almarza N.G., Bresme F., G. Stell. // Phys. Rev. E, 1994, vol. 49 , p. 5169 .

5. Tavares J.M., Gama M.M.T., Teixeira P.I.C., Weis J.J., Nijmeijer M.J.P. // Phys. Rev. E, 1995, vol. 52, p. 1915.

6. Nijmeijer M.J.P., Weis J.J. // Phys. Rev. Lett., 1995, vol. 75, p. 2887; Phys. Rev. E, 1996, vol. 53, p. 591.

7. Chen K., Landau D.P. // Phys. Rev. B, 1994, vol. 49, p. 3266.

8. Evertz H.G., Landau D.P. // Phys. Rev. B, 1996, vol. 54, p. 12302.

9. Bunker A., Chen K., Landau D.P. // Phys. Rev. B, 1996, vol. 54, p. 9259.

10. Costa B.V., Costa J.E.R., Landau D.P. // J. Appl. Phys., 1997, vol. 81, p. 5746.

11. Landau D.P., Krech M. // J. Phys. Cond. Mat., 1999, vol. 11, p. R1.

12. Ido Y., Tanahashi T. // J. Phys. Soc. Jap., 1991, vol. 60, p. 466.

13. Akhiezer I.A., Akhiezer I.T. // Sov. Phys. JETP, 1984, vol. 59, p. 68.

14. Akhiezer I.A., Akhiezer I.T. // Sov. Phys. Solid State., 1987, vol. 29, p. 48.

15. Mryglod I.M., Tokarchuk M.V., Folk R. // Physica A, 1995, vol. 220, p. 325.

16. Mryglod I.M., Folk R. // Physica A, 1996, vol. 234, p. 129.

17. Burden R.L., Faires J.D. Numerical Analysis. 5th ed. Boston, PWS Publishing, 1993.

18. Krech M., Bunker A., Landau D.P. // Comput. Phys. Commun., 1998, vol. 111, p. 1.

19. Swope W.C., Andersen H.C., Berens P.H., Wilson K.R. // J. Chem. Phys., 1982, vol. 76 , p. 637.

20. Frenkel D., Smit B. Understanding Molecular Simulation: from Algorithms to Applications. New York, Academic Press, 1996.

21. Allen M.P., Tildesley D.J. Computer Simulation of Liquids. Oxford, Clarendon, 1987.

22. Tuckerman M., Berne B.J., Martyna G.J. // J. Chem. Phys., 1992, vol. 97, p. 1990.

23. Suzuki M., Umeno K. - In: Computer Simulation Studies in Condensed Matter Physics VI, edited by Landau D.P., Mon K.K., Schüttler H.B., Springer, Berlin, 1993.

24. Folk R., Mryglod I.M., Omelyan I.P. (unpublished).

25. Craknell R.F., Nicholson D., Parsonage N.G. // Mol. Phys., 1990, vol. 71, p. 931.

26. Duane S., Kennedy A.D., Pendleton B.J., Roweth D. // Phys. Lett. B, 1987, vol. 195, p. 216.

27. Mryglod I.M., Omelyan I.P. // Mol. Phys., 1995, vol. 84, p. 235.

28. Mryglod I.M., Omelyan I.P. // Mol. Phys., 1997, vol. 91, p. 1005.

29. Mryglod I., Folk R., Dubyk S., Rudavskii Yu. // Physica A, 2000, vol. 277, p. 389. 


\title{
Спін-динамічні розрахунки колективних збуджень у магнітних рідинах
}

\author{
І.П.Омелян ${ }^{1}$, І.М.Мриглод ${ }^{1,2}$, Р.Фольк ${ }^{2}$ \\ 1 Інститут фізики конденсованих систем НАН України, \\ 79011 Львів, вул. Свєнціцького, 1 \\ 2 Інститут теоретичної фізики, Університет м. Лінц, \\ Альтенбергштрассе 69, А-4040 Лінц, Австрія
}

Отримано 29 квітня 2000 р.

Пропонується новий підхід до дослідження динамічних властивостей спінових рідин у комп'ютерному експерименті. Підхід базується на формалізмі операторів Ліувілля для опису Гамільтонової динаміки у поєднанні з методом факторизації експонентних пропагаторів типу Сузукі-Троттера. У результаті розвинуто сукупність нових алгоритмів для числового інтегрування рівнянь руху за наявності як трасляційних, так і спінових ступеней вільності, які зберігають фазовий об'єм і $є$ часо-зворотніми. Показано, що ці алгоритми можуть використовуватися у комп'ютерних розрахунках із набагато більшими часовими кроками порівняно з тими, які притаманні стандартним прогнозокоректованим схемам. Це нам дало змогу вперше виконати прямі розрахунки динамічних структурних факторів типу спін-спін, спінгустина і густина-густина для Гайзенбергівської моделі ферофлюїду. Встановлено, що подібно до чистих рідин густинний спектр може бути описаний в термінах теплової та звукових мод. Водночас, подібно до спінових граткових систем,у феромагнітній фазі у поздовжньому та поперечному динамічних структурних факторах спін-спін як основний спостерігається спін-хвильовий пік. Окрім того, у поздовжньому динамічному структурному факторі спін-спін виявлено додатковий пік, поява якого передбачалася нами раніше [Mryglod I., Folk R. et al., Physica A277 (2000) 389]. Частотна позиція цього піку повністю збігається з частотою звукових збуджень, що відображає вплив рідинної підсистеми на спінову динаміку. Можливість поширення поздовжніх спінових хвиль на звуковій частоті слід розглядати як новий ефект, що потребує експериментальної перевірки.

Ключові слова: Гайзенбергівська рідина, спінова релаксація, колективні моди, симплектичне інтегрування, факторизації Сузукі-Троттера

PACS: $02.60 . C b, 75.40 . G b, 75.50 . M m, 76.50 .+g, 95.75 . P q$ 